\title{
Optimizing sterilized logistics in hospitals
}

\author{
Citation for published version (APA):
}

van de Klundert, J., Muls, P., \& Schadd, M. (2006). Optimizing sterilized logistics in hospitals. METEOR, Maastricht University School of Business and Economics. METEOR Research Memorandum No. 051 https://doi.org/10.26481/umamet.2006051

Document status and date:

Published: 01/01/2006

DOI:

10.26481/umamet.2006051

Document Version:

Publisher's PDF, also known as Version of record

\section{Please check the document version of this publication:}

- A submitted manuscript is the version of the article upon submission and before peer-review. There can be important differences between the submitted version and the official published version of record.

People interested in the research are advised to contact the author for the final version of the publication, or visit the DOI to the publisher's website.

- The final author version and the galley proof are versions of the publication after peer review.

- The final published version features the final layout of the paper including the volume, issue and page numbers.

Link to publication

\footnotetext{
General rights rights.

- You may freely distribute the URL identifying the publication in the public portal. please follow below link for the End User Agreement:

www.umlib.nl/taverne-license

Take down policy

If you believe that this document breaches copyright please contact us at:

repository@maastrichtuniversity.nl

providing details and we will investigate your claim.
}

Copyright and moral rights for the publications made accessible in the public portal are retained by the authors and/or other copyright owners and it is a condition of accessing publications that users recognise and abide by the legal requirements associated with these

- Users may download and print one copy of any publication from the public portal for the purpose of private study or research.

- You may not further distribute the material or use it for any profit-making activity or commercial gain

If the publication is distributed under the terms of Article $25 \mathrm{fa}$ of the Dutch Copyright Act, indicated by the "Taverne" license above, 
Joris van de Klundert, Philippe Muls, Maarten Schadd

Optimizing sterilized logistics in hospitals

$\mathrm{RM} / 06 / 051$

JEL code : C61, I12

\section{METE@R}

Maastricht research school of Economics of TEchnology and ORganizations

Universiteit Maastricht

Faculty of Economics and Business Administration P.O. Box 616

NL - 6200 MD Maastricht

phone : ++31433883830

fax : ++31433884873 



\section{Optimizing sterilized logistics in hospitals}

Joris van de Klundert, Philippe Muls, Maarten Schadd

Corresponding author: Joris van de Klundert

Full Professor of Value Chain Optimization

Department of Quantitative Economics

Faculty of Economics and Business Administration

Universiteit Maastricht

Po Box 6166200 MD, Maastricht

The Netherlands

jj.vandeklundert@ke.unimaas.nl

+31433883281

December 5, 2006 


\section{Optimizing sterilized logistics in hospitals}

This paper deals with the optimization of the flow of sterile instruments in hospital which takes place between the sterilization department and the operating theatre. This topic is especially of interest in view of the current attempts of hospitals to cut costs by outsourcing sterilization tasks. Oftentimes, outsourcing implies placing the sterilization unit at a larger distance, hence introducing a longer logistic loop, which may result in lower instrument availability, and higher cost. This paper discusses the optimization problems that have to be solved when redesigning processes so as to improve material availability and reduce cost. We consider changing the logistic management principles, use of visibility information, and optimizing the composition of the nets of sterile materials.

keywords: sterilized logistics, logistic design, outsourcing, optimization, complexity 


\section{Introduction}

Roughly speaking, the cost of health care in developed countries amounts to 10 percent [2] of Gross Domestic Product and is expected to increase, in absolute and in relative terms. Hence, health care costs place an increasingly heavy burden on national and individual budgets, and many developing countries are implementing policies to (stimulate health care providers to) cut costs.

There is a widespread believe that the cost effectiveness of health care can be improved. Carter [2] claims that a cost cut in health care by 10 to 20 percent is possible. For hospitals in the Netherlands, a recent report [1] written on behalf of the secretary of health care - identifies opportunities for improvement in logistics of goods and pharmaceuticals of 1 billion euros, and another 2 billion euro in patient logistics. This report also concludes that a 20 percent cost reduction is possible. The larger part of the cost reduction can be achieved by relatively straightforward measures, such as adopting uniform work processes, standardizing materials, quantity discounts, et cetera. In addition, better planning, and appropriate use of information technology are identified as valuable improvement opportunities.

In this paper, we explore opportunities for improvements in a specific flow of goods in hospitals, the flow of sterile instruments. The improvements are based on an aligned combination of improvements in work processes and information technology.

A typical Dutch hospital - which is not different from hospitals in other developed countries - has invested millions of euros in sterile instruments. On a national level, the investment in sterile equipment can be estimated to exceed 500 million euro. Moreover, central sterilization service departments (CSSD's), are capital intensive and, at a national level, employ thousands of people. Be it via taxes or via insurance payments, the expenses that come with the required availability of sterile instruments are paid by the customer, the patient.

The sterile logistics processes also incur high opportunity cost. In many hospitals, the CSSD is located near the Operating Theatre (OT), in a central position in the hospital. Of course, using the valuable space the CSSD occupies for care and cure rather than for the secondary sterilization processes provides opportunities to improve the service to the customers.

The optimization of the logistics of sterilized items in hospitals has received little attention in scientific literature. A first paper by Fineman and Kapadia [3] deals with establishing appropriate stock levels, under the assumption of constant demand. Although their model is different, the issue is closely related to the topics dealt with in this paper. Fineman and Kapadia [3] consider partitioning the stock in two components: one part is the processing stock, which is kept because of the replenishment costs. The other component consists of replacement stock; stock for unanticipated use, which in their view can be due to instrument wear, instruments being lost or damaged, et cetera.

To the best of our knowledge, there are no later works on optimization of 
sterile logistics in the scientific literature. Perhaps, the lack of scientific interest must be explained by the lack of priority given to controlling the cost of the secondary process of sterile logistics by hospital themselves. Instead the focus has been on its reliability. The sterilization process may appear unimportant as long as it is well functioning, it is nevertheless a critical process. If contaminated instruments are not cleaned and sterilized well, they may cause serious infections on other patients. Hence the quality of the process is of prime importance. Similarly, if a particular instrument is needed in a life saving emergency operation, but not available, bad logistics may endanger patient lives. This focus on quality and availability has apparently long overshadowed cost efficiency.

As mentioned before, hospitals are currently under pressure to cut costs. Moreover, it is widely recognized that cost reductions in secondary processes free money to improve the primary processes of patient cure and care. Consequently, attention for optimizing the logistic processes involved in sterile logistics, centered around the CSSD and the OT, has increased. In particular, there is much attention for outsourcing of the CSSD. Examples of such initiatives can be found in The Netherlands, and Belgium [5].

In principle, a party specializing in sterilization services may attain the following cost reductions. It may attain a scale, and therefore a cost level, that is not attainable by single hospitals. Second, specialization may lead to higher process quality. Thirdly, personnel cost may be lower than for a hospital.

In whatever form, placing the CSSD at a distance, or even changing the logistic principles of the sterile logistics, entails the risks of lowering sterile item availability and increasing cost, rather than reducing them. Whether the potential benefits are realized, depends on the extend to which the logistic design and operation are optimized. This paper deals with these optimization problems.

Before we start the analysis however, let us spend a few words to clarify the current information technology situation. Of course, the demand for sterile instruments in the OT is determined by the surgeries taking place, some of which may be emergencies, and some of which may be planned. Most hospitals enter information on planned operations into the Hospital Information System (HIS). However, it is not uncommon for CSSD activities to be executed and planned without making use of this planning information. Moreover, many hospitals don't have information on the whereabouts of sterile instruments during the day, and hence cannot use this information in the planning and execution of the sterilization activities.

In Section 2 we will start the analysis by modelling the sterile logistics, and considering several logistic principles to manage the flow of sterile goods. Gradually we will move towards a more demand triggered process, and recalculate optimal inventory levels. Section 3 addresses the basic, static, optimization problems arising in this setting. The resulting cost minimization problems involve transportation and inventory costs. The presented problem formulations are akin to lot sizing and transportation problems, and are shown to be solvable 
Figure 1: Insert figure 1 here

in polynomial time by dynamic programming techniques. Section 4 extends these results to dynamic problem settings, and in particular addresses the value of real time information. Section 5 deals with the issue of optimally composing nets of sterile items, so as to reduce overall logistics costs. We address the complexity of several problem variations, and show a general version of the problem to be NP-hard. We subsequently formulate it as a mixed integer linear programming problem, which appears to be time consuming to solve.

\section{Basic Logistic Design}

A rudimentary design for sterile logistics and subsequent improvements is presented in [5], [6]. For ease of exposition (and since the aforementioned publications are in Dutch), we now briefly repeat the presented design and its improvements (see also Figure 1). We consider the flow starting from the sterile storage of the operation theatre. Here the sterile instruments are placed in stock. They are not stocked individually here, but grouped in nets. Typically, all items in one net are exactly the items needed for a particular surgery. However in general this need not be the case. It may be the case that the content of a net is more general, so as to apply to several types of surgery, or that one type of surgery requires nets of distinct types.

Shortly before an operation, the required nets are taken from the storage, put onto a cart, and this cart will be taken to the required OT room. During the operation, the sterile items, whether they are used or not, will become contaminated. When the surgery is finished, all materials will be brought to the contaminated storage of the OT, from where they are taken to the goods receipt of the CSSD. There they are dismounted, disinfected, perhaps precleaned, and subsequently put into the washing machines. After washing, the materials are regrouped to form nets. The nets are put into the autoclaves where the sterilization takes place. Once sterilized, the nets are placed in the sterile storage of the CSSD. From there they are brought to the sterile storage of the OT, which completes the closed loop.

Typically, usage plus replenishment takes more than half a day, even when the CSSD is next to the OT. Therefore, we assume throughout this paper that when the CSSD is outsourced, sterile nets can be used only once per day. Of course exceptions are possible, and careful planning may utilize those possibilities, but we disregard this possibility for ease and clarity of analysis.

The reader may notice that the flow of sterile instruments forms a closed loop. Within this closed loop, the point of consumption of the sterile instruments is the OT, and hence the utility of the material is highest when it is in

the sterile storage of the OT. An effective logistic control principle is therefore to replenish all items to this sterile storage of the OT as quickly as possible. 
In reality, a basic control strategy is thus to process all instruments that are taken from the sterile storage of the OT through the entire loop in one day, so that at the end of the day, they are again in the sterile storage of the OT. This basic control principle has several disadvantages. First of all, it requires maximum storage capacity at a place in the hospital, near the OT, where space is most needed for the primary process. Second, it may involve working extra hours by the CSSD for materials that are not needed the next day. Thirdly, it may incur unnecessary transportation. In redesigning the logistic process, there are some physically implied restrictions. Disinfection and cleaning of material cannot be postponed without limits. Processing of contaminated material must start reasonably quickly after it has been used.

In the example below, we present a basic model to analyse the presented logistic design and alternatives. In the analysis, we consider weekly costs. The weekly costs have three constituents, namely the transportation costs, the OT storage costs, and the instrument costs. The transportation costs are linear in the number of transports to the OT. The storage costs at the OT are linear in the required OT storage space. For ease of analysis we assume that the storage space of a net is proportional to the number of instruments it contains. Finally we consider the instrument unit cost which model the cost incurred by usage, handling, and sterilization of an instrument. The instrument unit cost are linear in the number of times an instrument is used. These three types of costs are the cost most important to the hospital when outsourcing the CSSD. It is not hard to see that when outsourcing the CSSD, transportation costs go up, while at the same time storage costs at the OT might go down. The instrument unit cost will come into play in a later stage.

Example 1 This example considers a case in which there are 5 different operation types $\{A, B, C, D, E\}$. There will be dedicated nets of instruments for each of the operation types. The operations of each of the operations require instruments of the eight instrument types $\{a, b, c, d, e, f, g, h\}$. The requirements are as follows:

1. Operation $A:(a, f, g)$

2. Operation $B:(b, f, g)$

3. Operation $C:(c, g)$

4. Operation $D:(d, h)$

5. Operation $E:(e, h)$

Planned operations take place according to the following weekly schedule:

1. Monday morning: $3 A, 6 D$

2. Monday afternoon: 3B: $6 D$ 
3. Tuesday morning: $3 A, 6 D$

4. Tuesday afternoon: 3C: $6 D$

5. Wednesday morning:: $1 C, 1 E$

6. Wednesday afternoon: $1 B: 1 E$

7. Thursday morning: $3 C, 6 E$

8. Thursday afternoon: 3B: $6 E$

The cost parameters are set as follows:

1. transportation cost (per transport) $=40$;

2. instrument usage cost (per instrument) $=1$;

3. storage costs (per capacity unit) $=9$;

In the above example, the basic strategy described above requires that all sets are being kept in storage simultaneously at the OT. Since sets can be used only once per day, the schedule implies that 3 sets for each of $A, B$ and $C$, and 12 sets for each of $D$ and $E$, in total 33 sets must be kept in storage simultaneously. For the moment we ignore the idea of keeping extra nets to cope with unplanned activities.

The capacity requirements for the sets are one unit per instrument, yielding a capacity requirement of 3 for nets of type $A$ and $B$, and 2 for the other nets. Thus total storage costs amount to $((3 \times 3)+(3 \times 3)+(3 \times 2)+(12 \times 2)+$ $(12 \times 2)) \times 9)=(72 * 9)=648$. Transportation is required once per day, for 4 days, yielding a transportation cost of $4 \times 36=144$. Finally, the instrument costs amount to 129, the number of instruments required to execute the weekly schedule. Hence, we have:

- transportation cost (per transport) $=160$;

- instrument usage cost (per instrument) = 129;

- storage costs (per capacity unit) $=648$;

- Total cost: 937

If we assume that the setting in which the sterile storage of the OT yields a transportation costs of zero, this setting still yields a total costs of 921-160= 761. Hence this 761 could serve as the reference cost. 
Thus, while continuing to neglect unplanned use of sterile sets for the time being, the initial effect of outsourcing the CSSD is an increase in total costs of 160 being the transportation costs. There are two underlying assumptions. First of all that transportation of contaminated sets from OT to the outsourced CSSD can be taken care of by the transportation devices that do the delivery, or at least without incurring extra costs. Second, that the storage costs remain unaltered. In fact, when outsourcing the CSSD, storage costs at the CSSD may increase to keep sterile sets in storage, whereas storage capacity in the hospital may decrease. In subsequent computations, we assume that there is an opportunity costs involved for every unit of storage costs that is in the hospital instead of outside of it. Hence, each unit of storage capacity that we eliminate from the hospital has a certain value.

We conclude that in the deterministic setting considered so far, the initial effect of outsourcing will be that the transportation times and costs increase. In a less stylized setting, where part of the surgeries are unplanned, e.g. emergency cases, some safety stock is required, and the amount of safety stock goes up as the lead time goes up due to longer transportation times. This incurs extra material costs. Van de Klundert et al. [6] report findings that roughly indicate a single unity increase in stock levels, on a real life case, if priority replenishments can be delivered within 2 hours, which is quite a mild assumption. Thus, without further modifications, outsourcing leads to higher costs. We now present logistic improvements which utilize the possibility to reduce storage space inside the hospital.

A first change in logistic design arises when part of the inventory that is currently kept at the sterile storage of the OT is moved to the sterile storage of the CSSD. However, this change requires to choose an inventory replenishment strategy for sterile storage at the OT. Most of the theory on replenishment strategies stems from nondeterministic models. We will dive deeper into the difficulties encountered in nondeterministic settings in later sections. The next section first strives to minimize total costs by jointly optimizing transportation and holding costs. This deterministic setting is akin to other deterministic replenishment models such as Lot Sizing models, and will also be solved using dynamic programming techniques.

\section{Deterministic Optimization}

The models in this section explicitly assume all surgeries and the resulting sterile instrument usage are completely predictable. In this case, sterile storage at the OT is not required. It is possible to deliver sterile items from the CSSD sterile storage just in time before the surgery begins. When transportation costs are high, this Just In Time design may lead to a cost increase. On the other hand, a cost reduction might be attainable when using a small sterile storage at the $\mathrm{OT}$, to reduce the number of required transportations (but with an increase in storage costs).

Notice that in our basic model storage costs at the sterile storage of the OT 
depend on the capacity of the storage, not on the inventory levels. (Nothing is to be gained from reducing inventory levels at the OT, if it leads to higher inventory levels in another part of the closed loop process.) Consequently, minimization of the transportation costs for a given OT schedule, takes the sterile storage capacity at the OT as an input parameter. We now model the resulting problem mathematically.

As a first modelling step we notice that any reasonable delivery schedule can be assumed without loss of generality to deliver instruments in the order in which their corresponding operations are scheduled. Moreover, the set of moments in time at which delivery occurs in an optimal solution can be discretized. Of course, materials are required at the start of an operation, and hence only the starting time moments need to be considered. However, in practice, OT rooms are scheduled in blocks which contain sequences of operations. (Of course blocks might consist of single operations). In this case, the delivery moments in an optimal solution can be assumed to occur at the start of a block.

In combination with the delivery order, this discretization yields that the joint optimization of the transportation costs and the storage costs, only needs to consider the volume of the instruments required in each block. (We shall see later that this is not valid when there is unplanned usage.) To see this, consider the case where a set of delivery moments is given. Then, the instruments delivered at each of these moments can easily be determined: simply deliver all instruments required for the blocks whose starting time falls between the current and the next delivery. Obviously, later delivery yields the solution to be infeasible. Earlier replenishment can only lead to an increase of required storage capacity. Thus, given the delivery moment, it is easy to decide when to deliver each of the required nets. Hence, the problem boils down to selecting a set of delivery moments which minimizes total costs.

Now, let $\left(t_{1}, \ldots, t_{m}\right)$ be the set of delivery opportunities for sterile nets as implied by the OT program. We let $Q_{i}=1$ if delivery takes place at $t_{i}$ and zero otherwise, for $i=1, \ldots, m$. The cost per delivery are denoted by $d$. The transportation capacity is modeled by $Q$, and $q_{i}$ is the volume delivered at $t_{i}, i=1, \ldots, m$. We denote by $S$ the storage capacity at the OT, by $e$ the storage costs per unit, and by $q_{i}, i=1, \ldots, m$ be the volume required for the sterile nets needed at time $t_{i}$. For $i=0, \ldots, m, h_{i}$ denotes the inventory position at time $i$. Now, the problem can be straightforwardly modelled as follows:

$$
\begin{gathered}
\min C=d \times \sum_{i=1}^{m} Q_{i}+e \times S \\
Q_{i} \times Q \geq q_{i} \quad i=1 \ldots, m \\
h_{0}=0 \\
h_{i}=h_{i-1}+q_{i}-c_{i} \quad i=1, \ldots, m \\
h_{i} \leq S \quad i=1, \ldots, m
\end{gathered}
$$


Figure 2: Insert Figure 2 here

$$
\begin{gathered}
q_{i}, h_{i} \geq 0 \quad i=1, \ldots, m \\
Q_{i} \in\{0,1\} \quad i=1, \ldots, m
\end{gathered}
$$

This model assumes that sterile nets which are used in the block immediately after delivery, never use storage capacity. Should one prefer to model that these nets do require storage, the storage capacity constraint 4 simply becomes

$$
h_{i}+c_{i} \leq S \quad i=1, \ldots, m .
$$

The case in which the capacity of the transportation vehicle is unbounded is a special case which can be modeled by setting $Q$ sufficiently large (e.g by setting $\left.Q=\sum_{i=1}^{m} c_{i}\right)$.

A graph representation for the resulting problem is depicted in Figure 2.

As is clear from Figure 2, the resulting problem is a special case of the fixed charge network flow problem, the decision version of which is in general known to be NP-Complete [4]. If capacity of the transportation device is bounded, and nets have non unit capacity requirements, the decision version of the delivery problem, can easily seen to be strongly NP-Complete, since it contains the 3 Partitioning problem (see e.g. [4]) as a special case. In the remainder we consider the case of unbounded transportation capacity. (In real life, the volume of the sterile sets is small enough to allow sets for blocks of an entire day to fit into a moderately sized truck, so that capacity of the transportation device is not limiting.)

The capacitated version of the problem in which $S$ is fixed can be easily solved. Since storage costs have become fixed at $e \times S$, costs $C$ are minimized by minimizing the number of deliveries. This is achieved by delivering a quantity of $S$ at the latest time moments $i$ possible, without incurring the solution to become infeasible. Thus, a first quantity $S$ is delivered at $t=0$, a second delivery at the earliest time moment $i$ for which $\sum_{k=0}^{i} c_{k}>S$, et cetera. The resulting delivery moments, and hence the resulting transportation costs, can thus be computed in $O(m)$ time. We denote this costs, for given storage capacity $S$ by $C(S)$. Now, let $S^{*}$ be a storage capacity such that

$$
C\left(S^{*}\right)=\min _{S} C(S)
$$

Moreover, for $1 \leq i<i^{\prime} \leq m$ define $c_{i i^{\prime}}=\sum_{k=i}^{i^{\prime}} c_{k}$. Then it is not hard to see that $S^{*}=c_{i i^{\prime}}$ for some $1 \leq i<i^{\prime} \leq m$. Hence the following straightforward procedure solves the problem of determining $A^{*}$ and a set of delivery moments minimizing $\left.C S^{*}\right)$.

1. For all $1 \leq i<i^{\prime} \leq m$ set $c_{i i^{\prime}}=\sum_{k=i}^{i^{\prime}} c_{k}$.

2. For all distinct values of $c_{i i^{\prime}}$ compute $C\left(c_{i i^{\prime}}\right)$ 
3. $C \rightarrow \min _{i, i^{\prime}} C\left(c_{i, i^{\prime}}\right)$.

The time complexity of this procedure is $O\left(m^{3}\right)$.

We now briefly consider a variation where storage costs are replaced by holding costs. In this model the costs of keeping inventory at the OT doesn't depend on $S$ but are modelled as $f \times h_{i}$ for each time interval between two consecutive delivery moments. The cost minimization problem can then be modelled as:

$$
\begin{gathered}
\min F=d \times \sum_{i=1}^{m} Q_{i}+f \times h_{i} \\
Q_{i} \times Q \geq q_{i} \quad i=1 \ldots, m \\
h_{0}=0 \\
h_{i}=h_{i-1}+q_{i}-c_{i} \quad i=1, \ldots, m \\
h_{i} \leq S \quad i=1, \ldots, m . \\
q_{i}, h_{i} \geq 0 \quad i=1, \ldots, m \\
Q_{i} \in\{0,1\} \quad i=1, \ldots, m
\end{gathered}
$$

Again, we denote by $F(S)$ the value of the optimal solution for this problem for given $S$. The problem of finding $F(S)$ once $S$ is given, can be solved by the following dynamic programming recursion. For every delivery opportunity $t_{i}$ we define $c(i, h)$ to be the minimum cost over all feasible replenishment strategies that result in an inventory level of $h$ at time $t$. We require $h_{0}=0$, and we set $c(0,0)=0$ and $c(0, h)=+\infty$ for $h=1, \ldots, S$. Now, $c(i, h)$ can be defined as follows:

$$
\begin{gathered}
c(i, h)=\min \left\{c\left(i-1, h+c_{i}\right)+e \times\left(h+c_{i}\right), d+\right. \\
\min _{q=1}^{Q} c\left(i-1, h-q+c_{i}\right)+e \times\left(h-q+c_{i}\right)
\end{gathered}
$$

Now let $S$ be the maximum stock level considered ( $S$ can be the storage capacity or any other upperbound on $h$ ). Then, the recursion yields a straightforward dynamic programming algorithm of $O(Q \times S \times m)$ time complexity for the problem of finding the optimal replenishment strategy. Notice that this time complexity is pseudopolynomial.

We conclude this session by demonstrating cost reductions by combinations of reducing storage capacity and corresponding transportation movements in the example below, which is a continuation of the previous example. 
Example 2 In the previous example, transportation took place once per day at the end of the day, and all used nets are returned to sterile storage at the OT. A first and major improvement is realized when delivering every day at the beginning of the day, the nets required for that day. This leads to a reduction in storage costs, while keeping transportation costs unchanged. Notice that since we assume that storage is only needed for nets used in blocks which start later than their delivery, storage is only needed for nets used in the afternoon. For the problem instance under consideration, this yields the following costs:

- transportation cost $=160$;

- instrument usage cost $=129$;

- storage costs $=\max (21+18+5+21) * 9=189$;

- Total cost: 478

Thus, keeping transportation costs unchanged, cost are reduced by around $50 \%$ simply by switching from logistic design. Further, the thus obtained solution yields lower cost than the original setting in which transportation costs were zero. (However, let it be noted that we don't claim the numbers in the example to be realistic or representative. They merely serve to demonstrate the effect of variations and improvements.)

Further cost reduction are possible, by optimizing transportation. As a simple example, first consider the case where we deliver twice per day. We obtain:

- transportation cost $=320$;

- instrument usage cost $=129$;

- storage costs $=0$;

- Total cost: 449

Finally, let us consider the case where we optimize storage costs and transportation costs simultaneously. For the example under consideration, the optimal delivery schedule is a schedule in which the nets for Wednesday morning are delivered Tuesday afternoon. Other days are delivered twice daily. Hence the resulting costs are: 
- transportation cost $=280$;

- instrument usage cost $=129$;

- storage costs $=36$;

- Total cost: 445

\section{Nondeterministic Optimization}

In this section, we dispose of our simplifying assumption that all required nets are known precisely in advance. The unpredictable nature of OT processes is of course an important feature, and if not dealt with appropriately, a matter of life and death. As mentioned before, the risks at stake are a likely explanation for logistic costs to have long been disregarded. Let us therefore consider the uncertainties that play a role.

1. Many of the patients arrive unexpectedly, and as emergency patients who need to be operated instantly;

2. It frequently happens that surgery of expected patients evolves in an unexpected manner, and hence that additional sterile equipment is needed;

3. Nets of sterile equipment may be incomplete, may become unsterile beforehand, et cetera;

4. Different surgeons use different nets, and the actual surgeon is not the planned surgeon.

If the variations and uncertainties regard the majority of the surgeries, and cannot be reduced, the basic logistic design to always replenish as quickly as possible to OT sterile storage may appear close to optimal. However, the larger the fraction of surgeries that can be planned, and are executed as planned, the more planning and monitoring information can be used effectively, in which case a change of logistic design may prove to reduce cost.

We start by considering some basic replenishment models, as they are currently being used in hospital practice. The two bin system is a simple and well known replenishment policy (see for instance [8]) which is also related to Just In Time systems. In a two bin system, the inventory level at the sterile storage of the OT is maximized to be two 'bins', per net. Nets are taken from bin one, until bin one is empty. Subsequently, nets are taken from bin two, until it is empty. Upon being emptied, bins are replenished so that when bin two is empty, bin one has been replenished and vice versa. Another simple policy used 
in practice is to always order the amount which has been used. More advanced ordering policies are for instance considered in ([3]).

Essentially, the only way to deal with unplanned usage of nets is to keep inventory for it at the sterile storage at the OT. Of course, if unplanned usage requires the same nets as the ones which are in inventory for planned usage, then the planned nets can be used. But these in turn might be needed shortly before replenishment arrives, or can arrive. Hence, safety stock is needed to anticipate unplanned usage. Safety stock must not only be kept, it might also have to be replenished. For the issue of replenishment, which of course should be aligned with the replenishment of planned usage, we propose four different strategies:

1. the original planning includes only deliveries for planned usage as computed using the aforementioned dynamic programming approach. Unplanned usage for the planning period has to be delivered from designated stock. This designated stock serves as a safety stock which should last through the entire planning period. This strategy doesn't require any information exchange on unexpected use or replanning.

2. The original planning includes both planned usage as well as expected demand for unplanned material usage. The optimal delivery schedule for this forecast is then computed using the dynamic programming approach described above. This approach is combined with an appropriately set initial safety stock level. Unplanned use does however not lead to changes in delivery schedule. Together with the safety stock, the current plan should suffice. As it was the case for the previous strategy, no (real time) information on usage is exchanged, and no replanning occurs

3. The idea is to schedule only delivery for planned usage of sterile nets using the dynamic programming approach presented above, and to guard against unplanned use by an initial safety stock. If stock reaches a level below the safety stock level, the transportation plan is dynamically reoptimized in such a way that immediate replenishment of net types which are below safety stock level is scheduled.

4. Initially the plan is to schedule delivery for planned usage and for expected demand for unplanned usage of sterile nets, using the dynamic programming approach presented above, and to guard against unplanned use by an initial safety stock. If stock reaches a level below the safety stock level, the transportation plan is dynamically reoptimized in such a way that immediate replenishment of net types which are below safety stock level is scheduled. In this reoptimization both planned usage and expected demand for unplanned usage are taken into account.

We compare the replenishment strategies under uncertainty, in a simulation environment, where the planned demand is fixed for a week, and in which there 
is a stochastic demand as well, which unfolds during the week. The first strategy deals very statically with the stochastic demand, it simply arranges sufficient safety stock. The second strategy only uses information that is a priori known, and safety stock. The third strategy reoptimizes using real time information. The fourth strategy uses a priori and real time information. Of course, other strategies are possible, but we limit our analysis to these basic strategies.

Let it be noted first though, that the nondeterministic setting is essentially different from the one discussed in the previous section, with respect to differences in net types. In the previous section, it was shown that the types of the nets didn't matter, only their volumes did. However, when keeping safety stocks, it is not the volume that counts, but also the type of the sets. Hence, the algorithms and models developed in the previous section are only valid in this nondeterministic setting if all nets are of a common type. This is for example the case when a same net is used for all types of surgery. Not a common situation in a general hospital, but a possible solution in specialized centers. The dynamic programming algorithms of the previous section can be extended to cases with multiple net types, but the state space of the dynamic programming methods grow exponentially with the number of set types [7].

The issue of composing nets will be taken into consideration after the continuation of our example below.

Example 3 In the comparison below, we say that during each morning and afternoon session of four hours, there is every hour a probability of 0.5 for unexpected changes in the OT schedule. For simplicity, we assume each unexpected usage to result in an extra use of one capacity unit of storage. Moreover, we have adapted the initial volume requirements of the Example 1 in such a way that the expected usage equals the usage implied in Example 1. Hence, the costs as computed in Example 2 can serve as a lowerbound for the minimum attainable cost in this non deterministic case.

The results presented below are average results over 50 simulations. We demand for each of the scenarios that safety stocks are such that stock outs never occur.

\section{No expected demand planned, frozen planning}

In this scenario, a total storage space of 35 is needed, and a safety stock of 26. The resulting total cost amount to 638 .

2. Expected demand planned, frozen planning In this scenario, a total storage space of 18 is needed, and a safety stock of 7 . The resulting total cost amount to 485 .

3. No expected demand planned, replanning In this scenario, a total storage space of 16 is needed, and a safety stock of 5. The resulting total cost amount to 469.

\section{Expected demand planned, replanning}


In this scenario, a total storage space of 14 is needed, and a safety stock of 3. The resulting total cost amount to 451.

Notice that the latter scenario induces a cost for the non deterministic case which is very close to the lowerbound of 445 which is the optimal solution in the deterministic case.

We conclude this section by considering the issue of minimizing stock levels, when taking differences between nets into account. To this purpose, we consider a simple delivery strategy. Planned net usage is scheduled using the dynamic programming algorithm, and unplanned usage is taken care of by taking extra stock which should suffice until the end of the planning period. Three solutions can be considered:

1. each operation type has its own net, and its own stock,

2. there is one net for all operations,

3. Each operation may require multiple nets, one dedicated net, and one net which serves more than one operation type, perhaps all operation types.

Obviously, the second solution entails a lower number of nets in stock than the first solution, leading to a potential decrease in storage costs, but the number of items per net must be higher, leading to an increase in storage cost, and in unit cost. The third solution is an unspecified combination of the first two. The example below already demonstrates that allows to easily construct solutions which outperform the first two simple solutions.

Example 4 Under the same settings as described before, the safety stock levels which ensure zero stock outs are: For type A, 2 nets, for type B, 3 nets, for type $C$, 3 nets, for type $D, 5$ nets, and for type E, 5 nets. In total this amounts to 18 nets. If a common net type is used for all operation types, a safety stock level of 8 nets suffices. Hence, in this example risk pooling effects [9] lead to a reduction of the number of sets in safety stock by more than 50 percent.

It then follows that if each type has its own safety stock, costs are the storage capacity cost per instrument, multiplied for each net type with the number of instruments times the required stock level. For the first solution, this amounts to $9 \times((3 \times 2)+(3 \times 3)+(2 \times 3)+(2 \times 5)+(2 \times 5))=9 \times 41=369$. For the second solution this amounts to $9 \times(8 \times 7)=504$. Notice that instrument unit cost are also much higher for this second solution.

A possible third solution is to put instruments $(g, h)$ into a common net, and the other instruments in separate ones. This leads to costs $9 \times((2 \times 2)+(2 \times$ $3)+(1 \times 3)+(1 \times 5)+(1 \times 5))+(2 \times 8))=9 \times 34=306$. When compared to the case of a single net per operation type, the expected extra instrument usage cost for this solution can be computed as follows. Per operation, one additional (obsolete) instrument is used, and the expected number of operations equals 58. Hence, in expectation, this solution yields a small improvement over the initial one. Further improvement is possible by putting the components of types $g$ and $h$ in separate nets. 
In the next section we consider the problem of finding an optimal net composition from a different angle.

\section{Optimizing the net composition}

In the previous sections the composition of nets was fixed, and hence storage cost, transportation cost, and instrument usage cost couldn't be decreased by changing the composition of the nets. In this section we will explore models for optimizing the composition of the nets. This net optimization problem will be called NOP. For ease and clarity of analysis we disregard transportation costs in this section, assuming that they are not affected by the net composition. Hence we only consider instrument usage costs, and storage costs. In addition to instrument related storage costs, we explicitly consider net related storage costs. This allows the model to also apply to cases in which the storage costs are not only dependent on the number of instrument in the net, but also on a constant term.

In the first solution proposed in the previous section every operation has its own dedicated net type, of which one net is used per operation. In this section, we will only consider the operations $A, B$ and $C$ from our previous example. For these operations, we need nets containing at least $(a, f, g)$ for operations of type $A$, nets containing at least $(b, f, g)$ for operations of type $B$, and nets containing at least $(c, g)$ for operations of type $C$.

This minimal solution: nets $(a, f, g)$ for operations of type $A$, nets $(b, f, g)$ for operations of type $B$, and nets $(c, g)$ for operations of type $C$, corresponds to the first solution presented in the previous section. It minimizes instrument usage cost, since it never happens that sets contain instruments which are not used. However from a viewpoint of storage costs, this method is not preferable. Many nets will be required, and since instruments are not used as frequently as possible, many instruments are needed as well.

The second solution presented in the previous section uses one net type for all 3 operations: nets containing $(a, b, c, d, e)$. This leads to an increase in instrument usage costs. The number of nets is as low as possible, yielding lower net storage costs costs, but instrument storage costs might still be high since even rarely used instruments are present in every set.

As mentioned before, one might expect that in between these extremes there exist better solutions, in which several operation types share a net type. For example, a net type $(a, b, f, g)$ for operation types $A, B$, and net type $(c, g)$ for operation type $C$. A further improvement is yet possible, by using more than one net per operation. For instance, a standard net $(f, g)$ can be composed, which is used for every operation type. We now give a mathematical programming formulation for a basic version of the net composition problem, and discuss its complexity. Variation and extensions can be found in [10].

The (additional) notation used to define the problem mathematically is as follows. We define a set of nets $j=1, \ldots, n$, as before, index $i=1 \ldots, m$ refers to the planning period. We use $k=1, \ldots, K$ to refer to the operations, and $l=$ 
$1, \ldots, L$ to refer to the sterile instruments. Finally, index $t, t=1, /$ ldots, $T$ refers to the instrument types. Basically, the problem boils down to deciding which materials go into each of of the nets, and to select nets for each of the operations such that each required material is in one of the selected nets. The model assumes that all instruments are unique. Moreover it assumes that instruments of a same type are indexed consecutively. Disregarding the objective function for the moment, a first natural formulation, uses the following decision variables:

- $M_{j l}=1$ if net $j$ contains instrument $l$ where $j=1, \ldots, n, l=1, \ldots, L, 0$ otherwise,

- $Z_{j i k}=1$ : net $j$ is used at day $i$ for operation $k, j=1 \ldots, n, i=$ $1, \ldots, m, k=1, \ldots, K, 0$ otherwise

Moreover, we introduce the following the parameters:

- $P_{l}$ : Instrument storage costs for instruments of type $t, t=1, \ldots, T$,

- $H$ : Net storage costs

- $S_{t}$ : Instrument usage cost for instruments of type $t, t=1, \ldots, T$

- $m_{t s}$ : The lowest index for instruments of type $t, t=1, \ldots, T$,

- $m_{t e}$ : The highest index for instrument of type $t, t=1, \ldots, T$,

- $N_{k t}$ : The number of instruments of type $t, t=1, \ldots, T$ needed for operation $k, k=1, \ldots, K$

The following constraints suffice to model the solution space.

$$
\begin{gathered}
\sum_{j} M_{j l} \leq 1 \quad l=1, \ldots, L, \\
\sum_{k} Z_{j i k} \leq 1 \quad i=1, \ldots, m, j=1, \ldots, n, \\
\sum_{j}\left\{Z_{j i k} \times \sum_{l=m_{t s}}^{l_{t e}} M_{j l}\right\} \geq N_{k t} \quad k=1, \ldots, K, i=1, \ldots, m, l=1, \ldots, L
\end{gathered}
$$

The first constraint models that an instrument can be in at most one net. The second constraint models that a net can only be used once per day. The third constraint models that if $N_{k t}$ instruments of type $t$ are needed for operation $k$, then, the sum over all nets used for this operations, of instruments of type $t$ is at least $N_{k t}$. This third constraint is not linear. It can be replaced by a set of linear constraints as follows. Let variable $Z_{j i k l}=1$ if instrument $l$ is contained 
in set $j$ and set $j$ is used for operation $k$ at day $i$, and 0 otherwise. We replace constraint 21 by four linear constraints.

$$
\begin{array}{r}
\sum_{j} \sum_{l=m_{t s}}^{m_{t e}} Z_{j i k l} \geq N_{k t} \quad i=1, \ldots, m, k=1, \ldots, K, t=1, \ldots, T, \\
Z_{j i k l} \leq M_{j l} \quad j=1, \ldots, n, i=1, \ldots, m, k=1, \ldots, K, l=1, \ldots, L \\
Z_{j i k l} \leq Z_{j i k} j=1, \ldots, n, i=1, \ldots, m, k=1, \ldots, K, l=1, \ldots, L \\
Z_{j i k l} \geq Z_{j i k}+M_{j l}-1, j=1, \ldots, n, i=1, \ldots, m, k=1, \ldots, K, l=1, \ldots, L
\end{array}
$$

The first of these constraints ensures that sufficient instruments are assigned to each operation, as did (21). The next contraint models that an instrument $l$ from net $j$ can only be used for an operation if $M_{j l}=1$, i.e. it is indeed in the net. Next, we model that if an instrument $l$ of net $j$ is used for an operation $k$ at day $i$, then $Z_{j i k}=1$, i.e. the net is used for the operation. The last one ensures that if a net is used for an operation, and the instrument is in the set, than we consider the instrument to be used as well, even if it is not needed. This constraint is required to appropriately model the objective function of the integer linear programming formulation below:

$$
\begin{gathered}
\min \sum_{j} \sum_{t} \sum_{j} l\left(M_{j l} \times P_{t} \sum_{j} Z_{j} \times H \sum_{j} \sum_{i} \sum_{k} \sum_{t} \sum_{j} l Z_{j i k l} \times S_{t}\right. \\
\text { s.t. } \sum_{j} M_{j l} \leq 1 \quad l=1, \ldots, L, \\
\sum_{k} Z_{j i k} \leq 1 \quad i=1, \ldots, m, j=1, \ldots, n, \\
\sum_{j} \sum_{l=m_{t s}}^{m_{t e}} Z_{j i k l} \geq N_{k t} \quad i=1, \ldots, m, k=1, \ldots, K, t=1, \ldots, T, \\
Z_{j i k l} \leq M_{j l} \quad j=1, \ldots, n, i=1, \ldots, m, k=1, \ldots, K, l=1, \ldots, L \\
Z_{j i k l} \leq Z_{j i k} j=1, \ldots, n, i=1, \ldots, m, k=1, \ldots, K, l=1, \ldots, L \\
Z_{j i k l} \geq Z_{j i k}+M_{j l}-1, j=1, \ldots, n, i=1, \ldots, m, k=1, \ldots, K, l=1, \ldots, L
\end{gathered}
$$

Now that $N O P$ is properly formulated, let us first consider its complexity.

Theorem 1 NOP is strongly NP Complete.

Proof 1 The polynomial ILP formulation presented above entails that NOP is in NP. Its completeness follows from a reduction from the NP-Complete 3dimensional matching problem (3DM). This problem is defined by Garey and Johnson [4] as followed:

INSTANCE: Three sets $A, B, C$ each containing $q$ elements, and a ternary relation $R \subseteq A \times B \times C$. 
QUESTION: Does $R$ contain a subcollection $R^{\prime} \subseteq R$ such that every element of $A, B$ and $C$ occurs in exactly one member of $R^{\prime}$ ?

Notice that the cardinality of $R^{\prime}=q$ and hence that without loss of generality $\|R\|>=q$. In the instances of NOP constructed in the reduction below $\|R\|$ corresponds with $L$, the number of instruments.

We polynomially construct an instance of NOP as follows. There are 3 days (i.e. $i=1,2,3$ ), and at each day $q$ operations have to be performed. The operations of day one are called $\alpha_{1} \ldots \alpha_{q}$, the operations of day two are called $\beta_{1} \ldots \beta_{q}$ and the operations of day three are called $\gamma_{1} \ldots \gamma_{q}$.

To determine which instruments are needed for every operation the following procedure will be applied. Each of the $L$ instruments, is of a unique type, and initially all operations need all $L$ instruments. For each $r \in R$ if $\alpha_{l} \in r$, then instrument $r$ is not needed for operation $l$ at day one, if $\beta_{l} \in r$ than instrument $r$ is not needed for operation $l$ at day two and if $\gamma_{l} \in r$ then instrument $r$ is not needed for operation $l$ at day three. The net storage cost is $L^{2}$ and instrument storage costs are 1.

Claim: The answer to $I$ is yes if and only if $I^{\prime}$ has a solution with value $q \times L^{2}+q \times(L-1)$.

Let $I$ be a yes-instance and let $R^{\prime}$ be the 3-dimensional matching for $I$. Now, for every $r \equiv\left(\alpha_{u}, \beta_{v}, \gamma_{w}\right) \in R^{\prime}$, the same net is used for operation $u$ at day 1 , for operation $v$ at day 2 and for operation $w$ at day 3. By definition, none of these operations need instrument $r$, and together they need all other instruments. Therefore the costs of the net consists of $L^{2}$ for net holding costs, plus $L-1$ for the instrument holding costs of the instruments contained in it. Because there are $q$ operations every day there have to be a total of $q$ nets and the total costs are $q \times m^{2}+q \times(m-1)$, completing the first part of the proof.

Now suppose $I^{\prime}$ has a solution $S^{\prime}$ of value $q \times L^{2}+q \times(L-1)$. Using that $q \leq L$, we derive that this solution cannot use more than $q$ nets because the costs would be at least

$$
\begin{gathered}
(q+1) \times L^{2}> \\
q \times L^{2} q \times(L-1)
\end{gathered}
$$

Any feasible solution uses at least $q$ nets, since there are $q$ operations each day, which yields that the solution uses exactly $q$ nets.

Thus each net is used every day, and for an arbitrary net, the three operations for which it is used will be called $u^{\prime}, v^{\prime}$ and $w^{\prime}$. By construction, the net needs to contain $L-1$ instruments if $\left(\alpha_{u^{\prime}}, \beta_{v^{\prime}}, \gamma_{w^{\prime}}\right) \in R$, and $L$ instruments otherwise. If $S^{\prime}$ has value $q \times L^{2}+q \times(L-1)$, then it must consist of $q$ sets of $L-1$ instruments. But, this implies that every net is used for a triple of operations $\left(u^{\prime}, v^{\prime}, w^{\prime}\right)$ corresponding to a triple $\left(\alpha_{u^{\prime}}, \beta_{v^{\prime}}, \gamma_{w^{\prime}}\right) \in R$. Since, the nets cover all $q$ operations, of each of the three days, the corresponding triples 
form a solution for $I$.

We have executed some computational experiments to solve instances of NOP using the ILP formulation presented above. Even small instances already required impractically large computation times using Cplex.

\section{Summary and directions for further research}

In the current quest for efficiency and effectiveness improvements in health care processes, redesigning the sterile logistics processes is frequently considered in practice. Despite this practical relevance, the problem of optimizing the loop of sterile logistics in a hospital has to the best of our knowledge hardly been studied in the scientific literature. This paper is therefore the first to explore various logistic designs, and models and methods for the resulting optimization problems, and to demonstrate the optimization potential.

The simplified and deterministic problems considered in Section 2 can be solved in polynomial time using dynamic programming techniques. Moreover, it is indicated that the potential improvements can be significant. The nondeterministic models in Section 3 are harder to solve. We explore and compare various strategies which use the findings of Section 2, and a priori and real time information. For these cases as well, we demonstrate that large improvements are possible by making appropriate use of optimization techniques. In the light of current advances in information technology, such as RFID (radio frequency identification) tags, the conclusion that appropriate use of technological advances yields a promising savings potential is justified. In fact is may well be that without the use of such technology, the potential benefits of more advanced logistic design can't be realized. Further practical research is required to confirm these findings.

Finally we identify and explore the issue of net composition. Again we demonstrate that there is a huge potential for optimization. We prove that a basic version of the problem is strongly NP-Complete. Moreover, it turns out that the presented integer linear programming formulation is very time consuming to solve. Nevertheless, we are in the opinion that this problem is interesting, and worthy of further research in the direction of solving realistic problem instances using heuristics as well as exact solution techniques.

\section{References}

[1] P. Bakker, Sneller Beter (in Dutch), Report, Dutch Ministry of Health.

[2] M. Carter, (2002), Diagnosis: mismanagement of resources, OR/MS Today, Vol 29, No 2. 
[3] S.J. Fineman, and A.S. Kapadia (1978), An Analysis of the Logistics of Supplying and Processing Sterilized Items in Hospitals, Computers and Operations Research, vol 5, 47-54

[4] M.R. Garey, \& D.S. Johnson, (1979), Computers \& Intractability, (W.H. Freeman \& Company),

[5] J.J. van de Klundert, (2005) Hoogervorst's optimaliseringsprobleem, (In Dutch) StatOR, Vol. 1\& 2, (2005) 8-12.

[6] J.J. van de Klundert, G.G. Van Merode, I.M.T. van Mulken, G. Tummers, , Het logistiek systeem van CSA en OK, een simulatiestudie (in Dutch), Maastricht University (2003),

[7] P. Muls, Materiaalstromen in een ziekenhuis: een simulatiestudie (in Dutch), Master's thesis, Maastricht University (2004)

[8] E. Silver, D. Pyke, R. Peterson, Inventory management and production planning and scheduling, (John Wiley 1998)

[9] D. Simchi-Levi, P. Kaminsky, E. Simchi-Levi, Designing \& Managing the Supply Chain ( McGraw-Hill, 2000),

[10] M. Schadd, The operation set problem, Bachelor Thesis, Maastricht University (2005). 


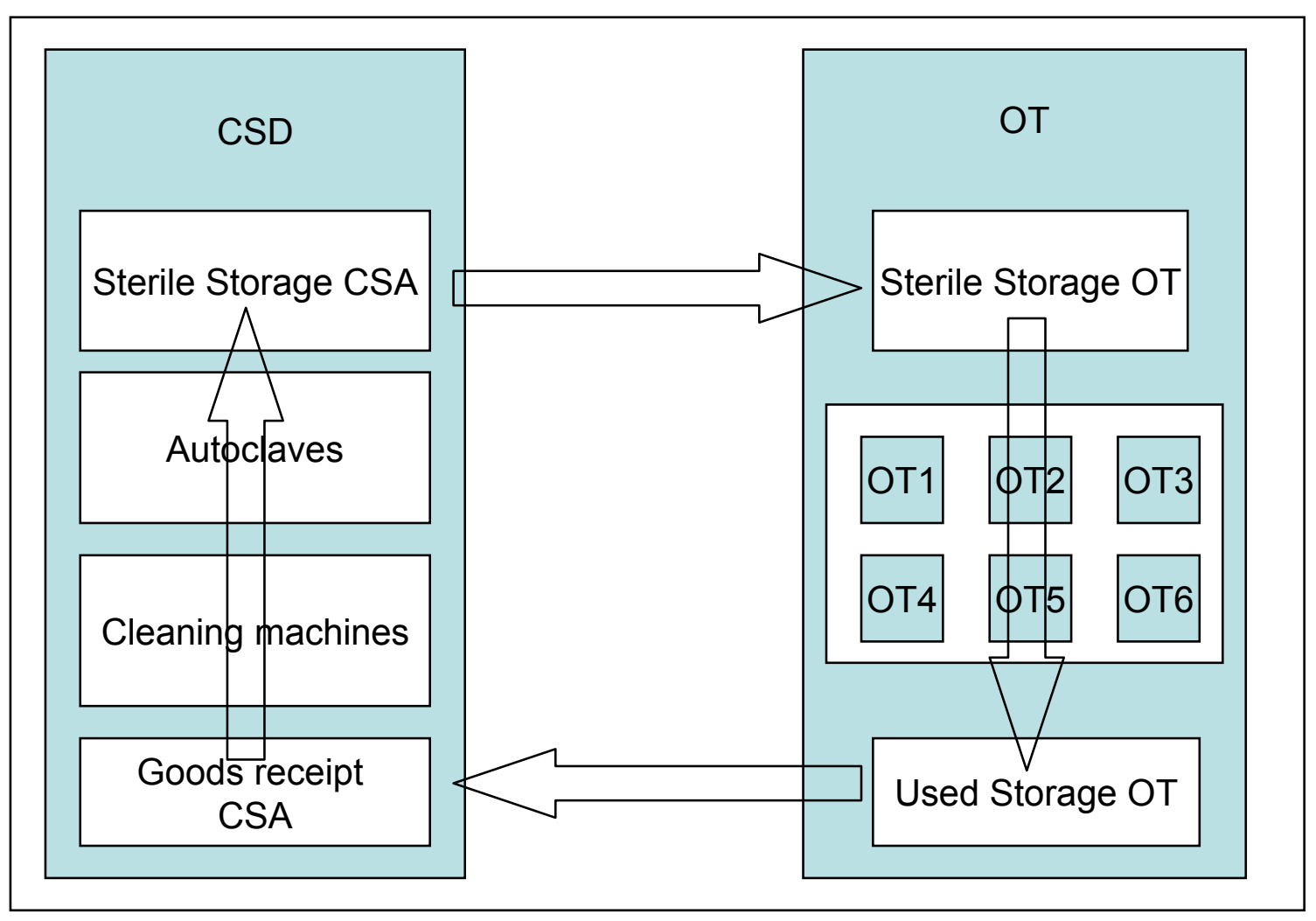




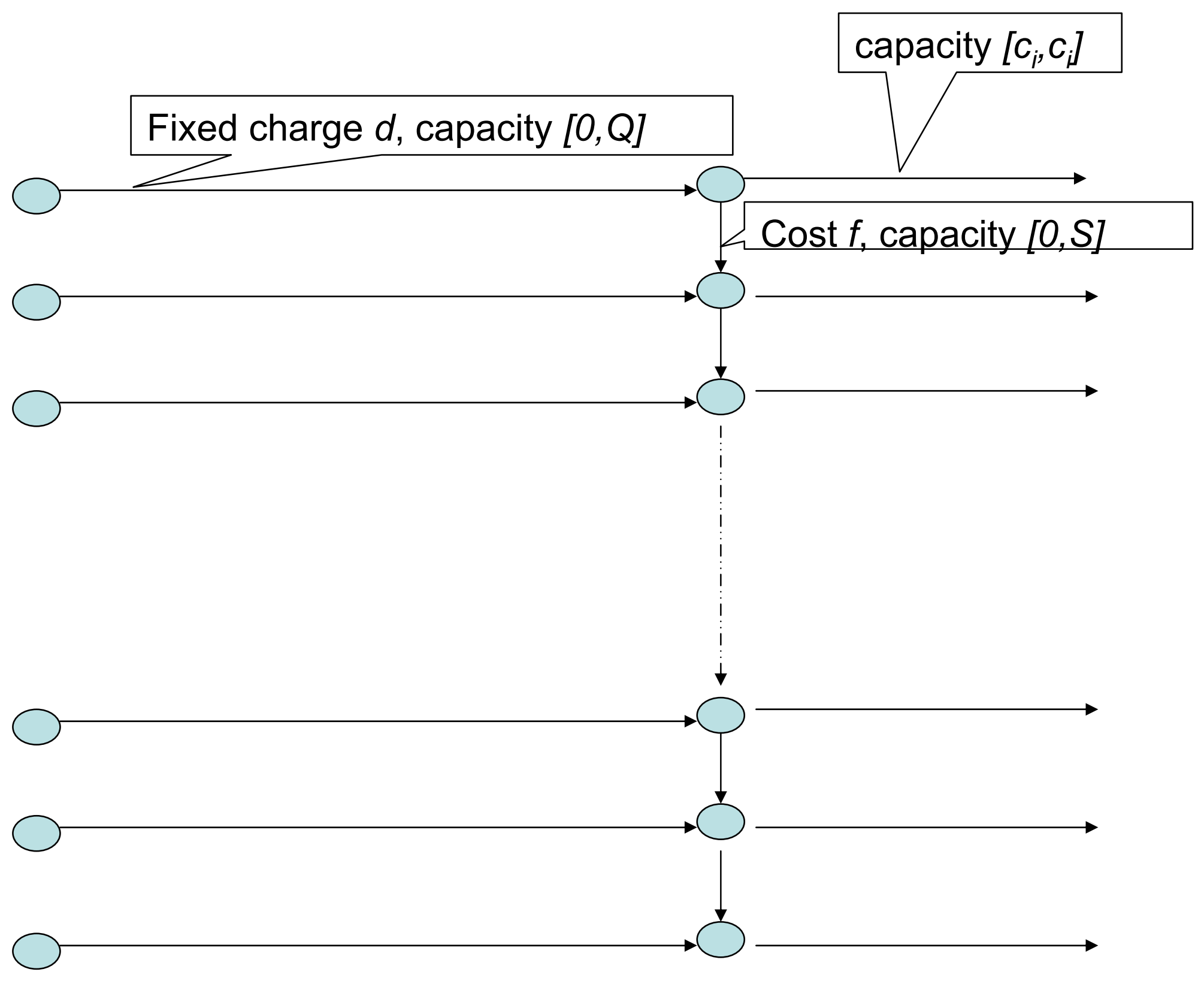

